\title{
Tools for the design of an inmotic system in the residential block of a five star plus hotel
}

\author{
José Ricardo Núñez Álvarez ${ }^{1}$, Israel Francisco Benítez Pina ${ }^{2}$, Danilo Mariano Carbonell Mestre ${ }^{3}$ \\ ${ }^{1,2,3}$ Universidad de Oriente - UO, Santiago de Cuba, Cuba. \\ Email: jricardo@uo.edu.cu, ibenitez@uo.edu.cu,danilo@emproy15.co.cu
}

Received: January $21^{\text {th }}, 2018$.

Accepted: February $15^{\text {th }}, 2018$.

Published: March $30^{\text {th }}, 2018$.

Copyright (02016 by authors and Institute of Technology Galileo of Amazon (ITEGAM).

This work is licensed under the Creative Commons Attribution International

License (CC BY 4.0).

http://creativecommons.org/licenses/by/4.0/ (c) (i) (3) Open Aceses:

\begin{abstract}
The purpose of this work is to select the tools for the design of an inmotic system to automate the housing blocks of hotel facilities in the province of Santiago de Cuba. This design leads to the obtaining of an intelligent building that favours the management and control of all its systems. It describes the networks used, their architecture and topology based on a distributed system where the horizontal structured cabling is achieved with twisted pair UTP and vertical cabling with fiber optic, as control elements, programmable logic controllers and different field elements are used for the measurement and execution of control actions where supervision is carried out through a SCADA on the basis of energy efficiency and comfort, safety, control and management, communications, accessibility and the building's life cycle.
\end{abstract}

Keywords: Inmotic, Intelligent Building, Energy Efficiency.

\section{Herramientas para el diseño de un sistema inmótico en el bloque habitacional de un hotel cinco estrellas plus}

\begin{abstract}
RESUMEN
Este trabajo tiene como propósito la selección de herramientas pertinentes para el diseño de un sistema inmótico para lograr la automatización de los bloques habitacionales de las instalaciones hoteleras en la provincia de Santiago de Cuba. Este diseño conduce a la obtención de un edificio inteligente favorecedor de la gestión y control de todos sus sistemas. En el mismo se describen las redes utilizadas, su arquitectura y topología basadas en un sistema distribuido donde el cableado estructurado horizontal se logra con par trenzado UTP y el cableado vertical con fibra óptica, como elementos de control se utilizan controladores lógicos programables y diferentes elementos de campo para la medición y ejecución de las acciones de control donde la supervisión se realiza a través de un SCADA sobre la base de la eficiencia energética y confort, la seguridad, el control y gestión, las comunicaciones, la accesibilidad y el ciclo de vida del edificio.
\end{abstract}

Palabras-clave: Inmótico, Edificio Inteligente, Eficiencia Energética.

\section{INTRODUCCIÓN}

En la sociedad actual es común apreciar y utilizar sistemas automatizados que cada vez abarcan mayor cantidad de sus esferas. Más, para una comprensión de tales sistemas, cuyo origen se enmarca en la segunda mitad del siglo XVIII, es preciso tener presente el concepto "automatización" y su característica principal de hacer funcionar un objeto o bien de forma semi-independiente del control humano.

Así, de tal concepto (automatización) surge lo que hoy conocemos como sistema automático, mediante el cual se efectúa y controla las secuencias de operaciones sin la ayuda de la actividad humana.

Como destacan Tiscornia y Defelipe, "la automatización se implementa mediante los distintos sistemas de control automáticos"[1]. Dichos sistemas se encuentran dispersos en varios campos: industrias, producción, servicios públicos, electrodomésticos, etc.

La importancia de contar con una comunicación efectiva y rápida; la necesidad de ahorrar energía; la disposición de los espacios y equipos; la posibilidad de dar un mayor ciclo de vida a un edificio; la seguridad, confort y comodidad de los 
trabajadores, han dado lugar al concepto de "edificios inteligentes".

- Según la definición dada por Percy Viego y un colectivo de estudiantes de la Facultad de Ciencias Económicas y Empresariales de la Universidad de Cienfuegos un edificio inteligente es aquel inmueble que desde su diseño incluye la automatización de sus sistemas, además del cuidado del medio ambiente donde se edificará, facilita su administración y mantenimiento, incentiva las labores diarias con instalaciones adecuadas y funcionales, permite obtener ahorros de energía en su operación, favorecer la operación y control con programas interrelacionados de todos los sistemas del edificio: hidrosanitarios, eléctricos, telecomunicaciones, seguridad, así como una flexibilidad para adecuaciones e innovaciones futuras. Debe asegurar un mantenimiento eficaz a bajo costo. Asimismo, garantizar una larga vida al inmueble [2].

Los edificios inteligentes se caracterizan porque hacen uso de la inteligencia artificial, proporcionan un ambiente de confort y seguridad para el aumento de la productividad y medios para el mantenimiento eficiente y oportuno minimizando los costos, además facilitan el cuidado del medio ambiente.

El progreso en paralelo de tres grandes áreas de la tecnología (telecomunicaciones, electrónica e informática) hizo posible en los años setenta el desarrollo de lo que actualmente se conoce como domótica [3].

Resulta imposible precisar una fecha concreta para el nacimiento de la domótica, ya que no se trata de un hecho puntual, sino de todo un proceso evolutivo que comenzó con las redes de control de los edificios inteligentes y se ha ido adaptando a las necesidades propias de la vivienda.

La palabra domótica, proviene de la unión de la palabra “domo" y el sufijo "tica". La palabra "domo" etimológicamente proviene del latín domus que significa casa, y el sufijo "tica" proviene de la palabra automática, aunque algunos autores también diferencian entre "tic" de tecnologías de la información y de la comunicación y "a" de automatización.

Este término proviene de la palabra francesa domotique, que la enciclopedia Larousse definía en 1988 como "el concepto de vivienda que integra todos los automatismos en materia de seguridad, gestión de energía, comunicaciones, etc."

Por lo que domótica se refiere al conjunto de técnicas utilizadas para la automatización de la gestión y la información de las viviendas unifamiliares [4].

- La domótica e inmótica expresan dos vertientes o ramas de la denominación edificio inteligente. La domótica referida a la adopción, integración y aplicación de las nuevas tecnologías informáticas y comunicacionales al hogar. La inmótica, es la aplicación de sistemas informáticos y nuevas tecnologías a grandes construcciones del sector terciario. De tal suerte, es la inmótica la que se dirige a la solución de la automatización en los hoteles.

- A partir de los años 90 Cuba se ha colocado entre los destinos mundialmente preferidos, lo que ha conducido al aumento de las capacidades hoteleras con la pretensión de lograr vincular la satisfacción del cliente con la eficiencia económica y en ello desempeña un rol determinante la tecnología que aun en las condiciones de país bloqueado se encuentra en constante cambio ante las más exigentes necesidades de los usuarios.

En este sentido, es política gubernamental para el Turismo priorizar el mantenimiento y renovación de la infraestructura turística y de apoyo. Aplicar políticas que garanticen la sostenibilidad de su desarrollo, implementando medidas para disminuir el índice de consumo de agua y de portadores energéticos e incrementar la utilización de fuentes de energía renovable y el reciclaje de los desechos que se generan en la prestación de los servicios turísticos.

- Sin embargo, de la literatura revisada y de la experiencia personal laboral especialmente obtenida como parte de una comisión designada para realizar el Estudio de Inicio de Operaciones que recorrió los principales hoteles de La Habana y Varadero en el 2015, incluido intercambios técnicos, pudo apreciarse que las soluciones dadas no responden a diseños de sistemas inteligentes.

- En tal sentido esta carencia determina este trabajo que busca identificar la importancia y potencialidades en el uso de los sistemas domóticos en la provincia de Santiago de Cuba.

- En la sección 2 se tratan los campos genéricos de las TIC, estos conforman los diferentes sistemas que definen un edificio inteligente, así como las características y aplicación de cada uno. La sección 3 brinda la arquitectura, clasificación y topología de las redes y se definen los medios de transmisión utilizados haciendo una breve descripción de cada uno y brindando los elementos seleccionados. En la sección 4 se detalla el SCADA, PLC y elementos de campo utilizados, definiendo el software de supervisión y control además se caracterizan y seleccionan los PLC y diferentes elementos de campo. A través de la sección 5 se enuncian las consideraciones finales y en la sección 6 se hace referencia a la bibliografía.

\section{CAMPOS GENÉRICOS DE APLICACIÓN DE LAS TIC EN EDIFICIOS INTELIGENTES}

Aquí se abordan los campos genéricos de aplicación de las TIC a la Edificación Inteligente tales como: eficiencia energética y confort, seguridad, control y gestión, comunicaciones, accesibilidad y finalmente aplicaciones específicas para la gestión de las fases del ciclo de vida del edificio [5].

\section{II.1 EFICIENCIA ENERGÉTICA Y CONFORT}

- Eficiencia energética: Estos sistemas de automatización permiten a los usuarios adaptar las condiciones ambientales estableciendo perfiles de consumos energéticos.

- Confort: Es necesario monitorizar y controlar las variables que lo afectan.

Aquí se logra la eficiencia energética y el confort en las habitaciones por medio del control de la temperatura a través de un termostato de temperatura digital que permite puntos de consigna mínimos y máximos, el control de la iluminación por medio de detectores de presencia que controlan los niveles de iluminación lo que conlleva a mayor eficiencia en el consumo eléctrico.

\section{SEGURIDAD}

La seguridad viene dada por la prevención, detección y solución de accidentes o imprevistos.

Existen sistemas de seguridad que protegen a las personas y los que protegen el patrimonio inmobiliario o bienes.

Para la seguridad de las personas se emplean:

- Sistemas de evacuación con apertura automática de salidas de emergencia.

- Redes de rociadores automáticos.

- Sistemas de detección y alarma para alerta de humo, fuego y fugas en las diversas instalaciones. 

equipadas.

- $\quad$ Sistemas de extracción de gases.

Para la seguridad y protección de bienes se emplean:

- Detectores específicos de movimientos, rotura de cristales y apertura de puertas.

- Sistemas de circuitos cerrados de televisión.

- Intercomunicaciones de emergencia.

- Control de accesos.

- Circuitos de ronda perimetral.

- Sistemas de seguridad informática.

Para la seguridad en la habitación se utilizan detectores de humo y contactos magnéticos en los marcos de puertas y ventanas que mantienen una vigilancia permanente contra posibles incendios e intrusos respectivamente.

\section{II.3 CONTROL Y GESTIÓN}

El sistema de control y gestión centralizada de las instalaciones generales es el de mayor y más extendida aplicación en las edificaciones de instalaciones con gran complejidad.

En este caso se utiliza el SCADA EROS, creado por la empresa SERCONI de la industria del Níquel en Holguín, Cuba, el cual permite configurar de manera sencilla gran número de variables, facilita el mando a distancia y el control desde la aplicación y gestiona eficientemente cada uno de los subsistemas que son responsables de las principales funciones del edificio (iluminación, climatización, seguridad, etcétera).

Este control y gestión se realiza desde el interior del edificio, específicamente desde el local central de automatización.

\section{II.4 COMUNICACIONES}

Las comunicaciones son el campo de aplicación con máximo protagonismo directo. Brinda apuntes esquemáticos y generales, sobre las telecomunicaciones en los edificios.

Para este caso se utilizan redes distribuidas de cableado estructurado donde se utiliza par trenzado UTP en el cableado horizontal y fibra óptica en el cableado estructurado vertical permitiendo velocidades de transmisión acorde a los estándares internacionales. Además, se utiliza televisión interactiva a través de pantallas profesionales táctiles.

\section{II.5 Accesibilidad}

Aquí se deben distinguir dos grupos principales en función del objetivo con que se enfoque en cada caso:

- Accesibilidad como tecnología: cuando la accesibilidad se centra en aplicaciones creadas para generar un determinado servicio al usuario, actuando como intermediaria entre la tecnología y el usuario.

- Accesibilidad como servicio: cuando la accesibilidad actúa directamente sobre el usuario, ofreciendo un servicio en sí mismo.

En este trabajo se tuvo muy presente la eliminación de las barreras arquitectónicas, teniendo la accesibilidad como tecnología lo que contribuyó a un diseño orientado a la mejora de la accesibilidad, haciendo especial hincapié en la accesibilidad

asociada a las Tecnologías de la Información y la Comunicación, como herramienta potencial de integración social.

\section{II.6 FASES DEL CICLO DE VIDA DEL EDIFICIO}

Este campo abarca las etapas de la vida de la edificación, en concreto su diseño, construcción y, finalmente, demolición y gestión de RCD (residuos de construcción y demolición).

En la actualidad, la construcción de edificios se ha convertido en una labor de creciente alcance, que pone en manos del diseñador el gran reto de conferir a su producto unas características de sostenibilidad cada vez más marcadas, que redunden en la eficiencia energética y en la reducción de las emisiones asociadas.

Las TIC deben proporcionar herramientas que gestionen de forma inteligente y eficiente todo el ciclo de vida de la edificación. Estas herramientas propician, además, entre otras actividades, la selección de la arquitectura, clasificación y tipología de las redes utilizadas.

\section{ARQUITECTURA, CLASIFICACIÓN Y TOPOLOGÍA DE LAS REDES. MEDIOS DE TRANSMISIÓN}

Con el avance de las nuevas tecnologías de la información y en particular el avance en el campo de la microelectrónica se han desarrollado métodos de conexión en los sistemas desde el punto de vista comercial. Existen varios tipos de redes, entre estas las que se dedican a la industria e informática que se dividen de acuerdo a su arquitectura, clasificación y topología. Para el intercambio de información en estas redes se utilizan los medios de transmisión.

La arquitectura de un sistema domótico o inmótico especifica el modo en que los diferentes elementos de control del sistema se van a ubicar [6].

Arquitectura Distribuida: Es una arquitectura basada en nodos, no existe un único elemento principal, sino que cada subsistema administra una tarea de control en particular y estos van relacionados directamente con los elementos básicos. Estos sistemas se comunican por medio de un bus, el que posee un protocolo de comunicaciones hecho en cada uno de los subsistemas con unas técnicas de direccionamiento definidas para mantener el intercambio de información entre los diferentes elementos. Ver figura 1a).

Arquitectura Centralizada: Aquí el control y supervisión de los elementos y componentes se deben cablear hasta un sistema de control en el edificio, el cual puede ser un computador personal o un autómata. Este sistema es el principal control ya que recibe y reúne la información de los sensores, toma las decisiones y se las envía a los actuadores para que realicen la tarea designada. Ante cualquier falla que presente deja fuera de servicio el sistema domótico en su totalidad y sus reconfiguraciones son muy costosas. Los elementos que la componen no necesitan módulos adicionales para el direccionamiento ni interfaces de comunicación para distintos buses. Ver figura $1 b$ ).

Arquitectura Descentralizada: Es una combinación entre los sistemas con arquitectura centralizada y distribuida. Está basada en una o varias unidades de control de gestión y uno o varios módulos receptores o actuadores. Es la arquitectura en que todos los sistemas son totalmente independientes en su funcionamiento, pero deben estar comunicados entre sí por medio de un bus compartido. Ver figura 1c). 


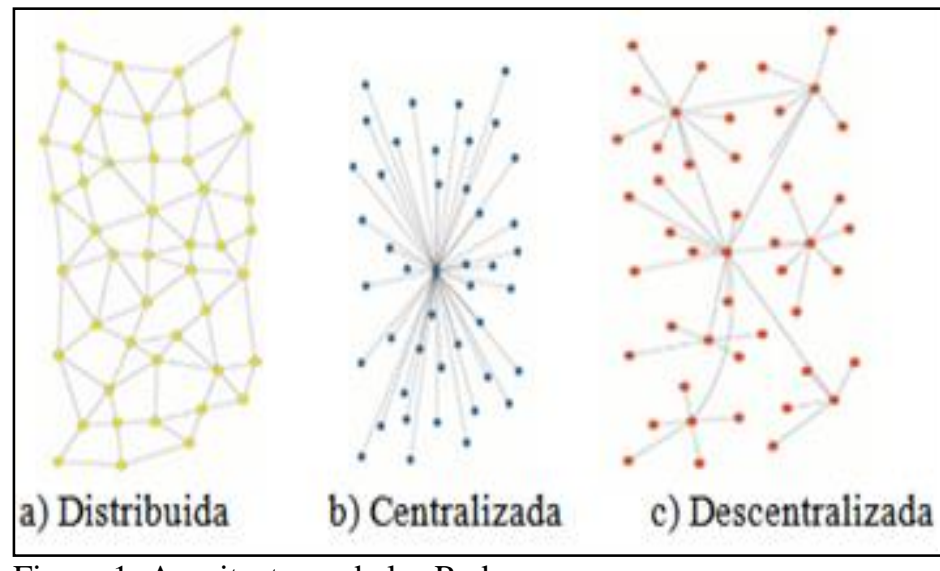

Figura 1: Arquitecturas de las Redes.

Fuente: Los autores, (2018).

En este artículo se utiliza una arquitectura de red distribuida que tendrá una estación de supervisión y mando central con una microcomputadora ubicada en el local central de automatización donde a través del bus se enlazan todos los elementos del sistema, cada elemento tiene una dirección IP.

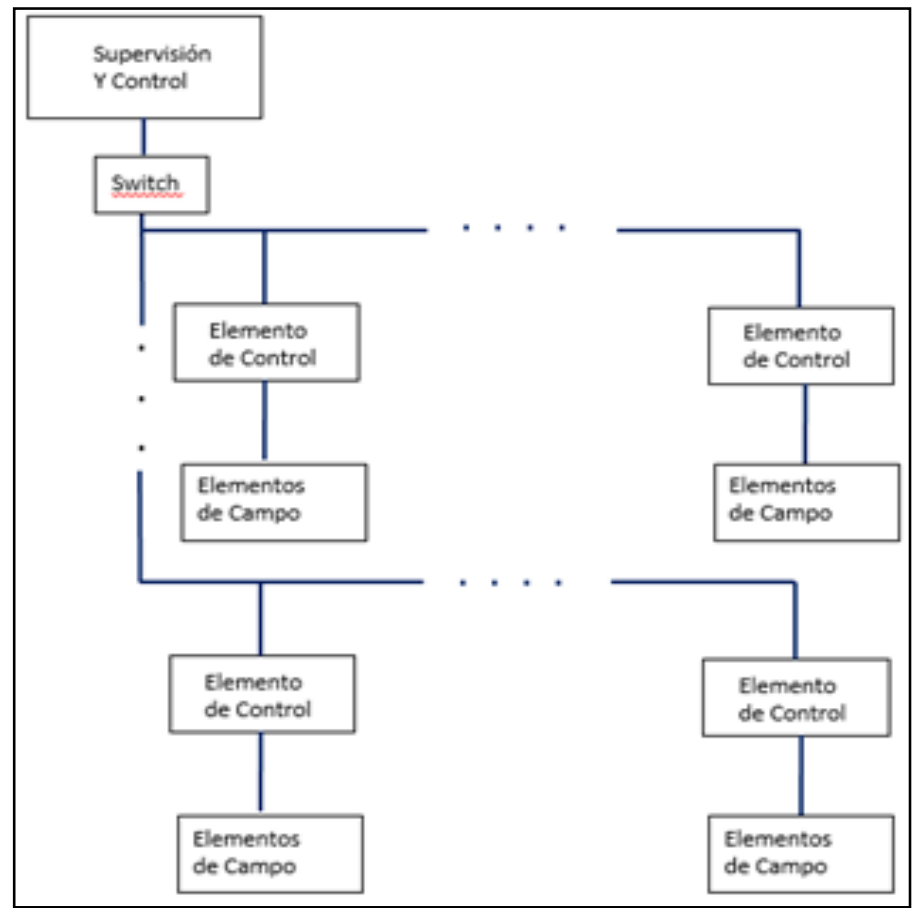

Figura 2: Diagrama en bloques de Red distribuida.

Fuente: Los autores, (2018).

\section{1 CLASIFICACIÓN DE LAS REDES}

Luego de realizar un estudio sobre las características de las redes industriales de automatización, del equipamiento utilizado y de los datos que van a circular por las mismas, se selecciona la Red Ethernet TCP/IP para lograr el cableado estructurado vertical (back bone), que enlaza el cuarto de control con los diferentes switch ubicados en los pisos habitacionales. Dicha red estará formada por fibra óptica en modo monomodo, la cual tiene un revestimiento o cladding de 125 micrómetros y un núcleo de 9 micrómetros a una velocidad de transmisión no inferior a 10.0 Gbps. Además, se utiliza el protocolo Modbus TCP/IP para lograr el cableado estructurado horizontal, comunicando el termostato de temperatura con pantalla LCD, que incluye el estado de funcionamiento (refrigeración, calefacción y ventilación), velocidad del ventilador, temperatura ambiente y punto de ajuste, con el autómata de la habitación en tiempo real a través de cable UTP de $4 \times 0.75 \mathrm{~mm}^{2}$. Este protocolo también se emplea en la interface de comunicación inteligente Acti 9 Smartlink para el control de los breakers termo magnético en las pizarras eléctricas utilizando el conector RS 485 [16][17].

Con la selección de este tipo de red se logra robustez, seguridad y fiabilidad de la información. Además, se obtiene un sistema modular, sostenible, actualizable, que puede implementarse por etapas, así como migrar a otras variantes tecnológicas similares o superiores. El autómata seleccionado Modicon M221 trae un puerto embebido de este tipo a través de conector RJ 45.

\section{III.2 TOPOLOGÍA DE LAS REDES}

La topología de red se define como la cadena de comunicación usada por los nodos que conforman una red.

La topología la determina únicamente la configuración de las conexiones entre nodos y no las distancias entre ellos, las interconexiones físicas, las tasas de transmisión y los tipos de señales.

La topología de las redes determina como están conectados los componentes en una instalación domótica (sensores, unidades lógicas de control, actuadores, etc.) respecto al medio de comunicación. Ver figura 3.

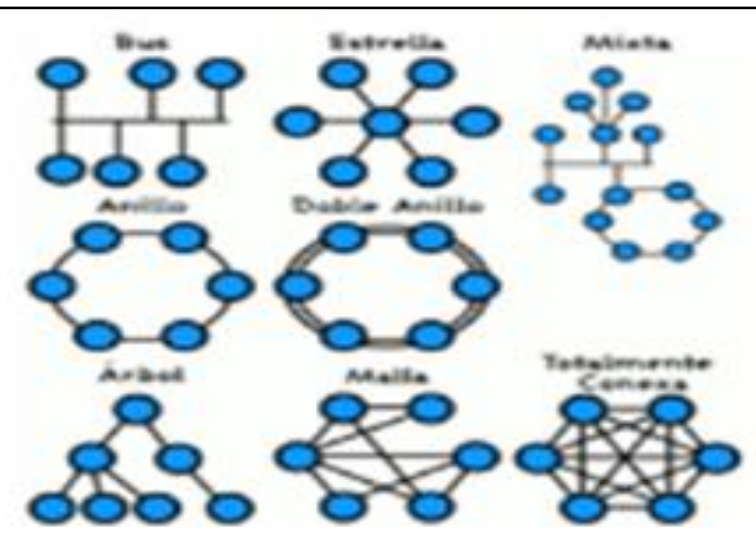

Figura 3: Diferentes topologías de Red.

Fuente: Los autores, (2018).

Aquí se utiliza una topología en bus en la que todas las estaciones están conectadas a un único canal de comunicaciones por medio de unidades interfaz y derivadores. Las estaciones utilizan este canal para comunicarse con el resto.

Se decide por esta topología en bus ya que con respecto a las otras tiene la facilidad en cuanto a añadir y quitar nodos al bus, además si un nodo tiene un fallo no afecta al resto del sistema como pasa con la estrella y el árbol, presenta menor costo en el cableado que otras estructuras, por ejemplo, la malla y permite realizar un sistema por etapas independientes.

\section{III.3 MEDIOS DE TRANSMISIÓN}

Los tipos de medios más utilizados para transmitir información en los sistemas domóticos son [8][9]:

- Sistemas que usan en todo o en parte señales que se acoplan y transmiten por la instalación eléctrica de baja tensión, tales como sistemas de corrientes portadoras. 
- $\quad$ Sistemas guiados que utilizan en todo o en parte señales transmitidas por cables específicos para dicha función, tales como cables de pares trenzados, paralelo, coaxial o fibra óptica.

- Sistemas (no guiados) que usan señales radiadas, tales como ondas de infrarrojo, radiofrecuencia o ultrasonidos.

En este caso se utiliza en el cableado estructurado horizontal par trenzado UTP de 100 ohmios y un ancho de banda de $100 \mathrm{Mhz}$ con una velocidad de transmisión no menor de 100 Mbps de 2x0.75 $\mathrm{mm}^{2}$ y una longitud máxima de $100 \mathrm{~m}$. En el caso del termostato de temperatura digital se utiliza cable par trenzado de $4 \times 0.75 \mathrm{~mm}^{2}$. En este tipo de cable dos conductores son entrelazados de forma helicoidal para suprimir las interferencias de fuentes externas y la diafonía de los cables adyacentes.

En el caso del cableado estructurado vertical (back bone), que enlaza el cuarto de control central con los diferentes switch ubicados en los pisos habitacionales, estará formado por fibra óptica en modo monomodo, la cual tiene un revestimiento o cladding de 125 micrómetros y un núcleo de 9 micrómetros a una velocidad de transmisión no inferior a $10.0 \mathrm{Gbps}$. Esta es un medio de transmisión inmune a las interferencias. Donde se transmite un sólo haz de luz por el interior de la fibra tiene un alcance de transmisión de $300 \mathrm{~km}$ en condiciones ideales, siendo la fuente de luz un láser. Ver figura 4.

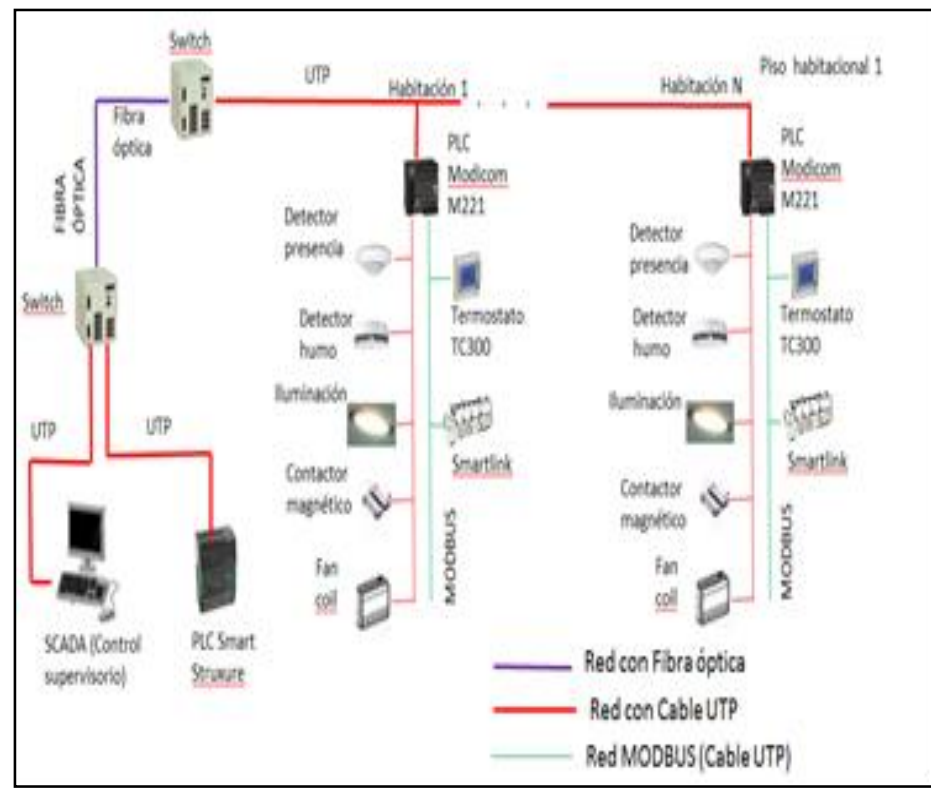

Figura 4: Esquema de comunicación horizontal.

Fuente: Los autores, (2018).

La categoría del cableado estructurado es $5 \mathrm{E}$ con una atenuación de $22 \mathrm{db}$ y una pérdida de retorno de $20.1 \mathrm{db}$. Todos los dispositivos de la red deben ser de categoría $5 \mathrm{E}$.

Como parte de los medios de transmisión se pueden tener en cuenta los switch, de los cuales se utiliza el TCSESM163F2SCO de Schneider Electric que se conecta por medio de conector RJ 45, de categoría 5E, con 14 puertos 10/100 BASE -TX y 2 entradas de fibra óptica monomodo 100 BASEFX (SCduplex).

Estos switch permiten el enlace entre el SCADA y los PLC en los diferentes pisos habitacionales.
IV. SCADA, PLC Y ELEMENTOS DE CAMPO. PANTALLA TÁCTIL

Un sistema SCADA es una aplicación o conjunto de aplicaciones software especialmente diseñada para funcionar sobre ordenadores de control de producción, con comunicación digital con los instrumentos y actuadores, e interfaz gráfica de alto nivel con el usuario (pantallas táctiles, ratones o cursores, lápices ópticos, entre otros) [10][18].

Un sistema SCADA está conformado por sistema de comunicaciones, unidad terminal remota (RTU), interfaz hombre-máquina (HMI), unidad terminal maestra (MTU) y transductores [7][8].

El término SCADA proviene de las siglas de "Supervisory Control And Data Acquisition", es decir, Control Supervisorio y Adquisición de Datos [19].

Como sistema de control supervisorio y adquisición de datos, SCADA, se utiliza el EROS. Creado para la industria del níquel, EROS es un sistema distribuido en el que sus diferentes componentes se interconectan a través de la Intranet Empresarial, siendo los componentes:

- Estaciones de medición.

- Estaciones de visualización.

- Servidores de reportes y servidores de tiempo.

- Un gran número de variables es posible configurar de manera sencilla con EROS. La configuración del sistema es en caliente, por lo que no necesita detener el proceso de supervisión para efectuar cambios en la configuración. Utiliza toda la potencia en edición de tablas y gráficos que posee el Microsoft Excel y lo combina con la posibilidad de insertar en celdas seleccionadas los valores que se necesiten de las variables del EROS. La capa de red permite integrar a los sistemas que están operando en diferentes máquinas, haciendo visibles para cualquier sistema las variables situadas en ordenadores remotos [12].

- EROS se escoge por encima de otros SCADAs, como One Supervisor de Omron o Vijeo Citect de Schneider, por su número prácticamente ilimitado de variables con procesamiento estadístico, además facilita el control de la disciplina tecnológica de forma rápida y segura, puede trabajar minimizado permitiendo mantener el sistema aun cuando se necesite hacer uso de otros programas como Word, Excel, etc.

- $\quad$ EROS puede procesar funciones lógicas y matemáticas programadas por el usuario, se comunica con los dispositivos a través de manejadores lo que aporta mayor flexibilidad y capacidad de adaptación a nuevos entornos, permite comunicación con autómatas SIEMENS, MODICON, NOVA, OMRON, SISCAL y otros, su fácil configuración en línea permite que usuarios no especializados, con orientaciones sencillas, puedan configurarlo.

En el supervisor descansa la responsabilidad de orientar o corregir las acciones que se desarrollan, por lo que se tiene una toma de decisiones sobre las últimas acciones de control por parte del supervisor, que recaen sobre el operario [10].

A través del SCADA propuesto se podrá contar con un monitoreo en tiempo real a distancia de las variables de las habitaciones y en caso de presentarse alguna anomalía o falla en una de ellas se estará en la capacidad de detectarlo de inmediato $\mathrm{y}$ tomar las acciones correctivas de acuerdo al problema planteado. 


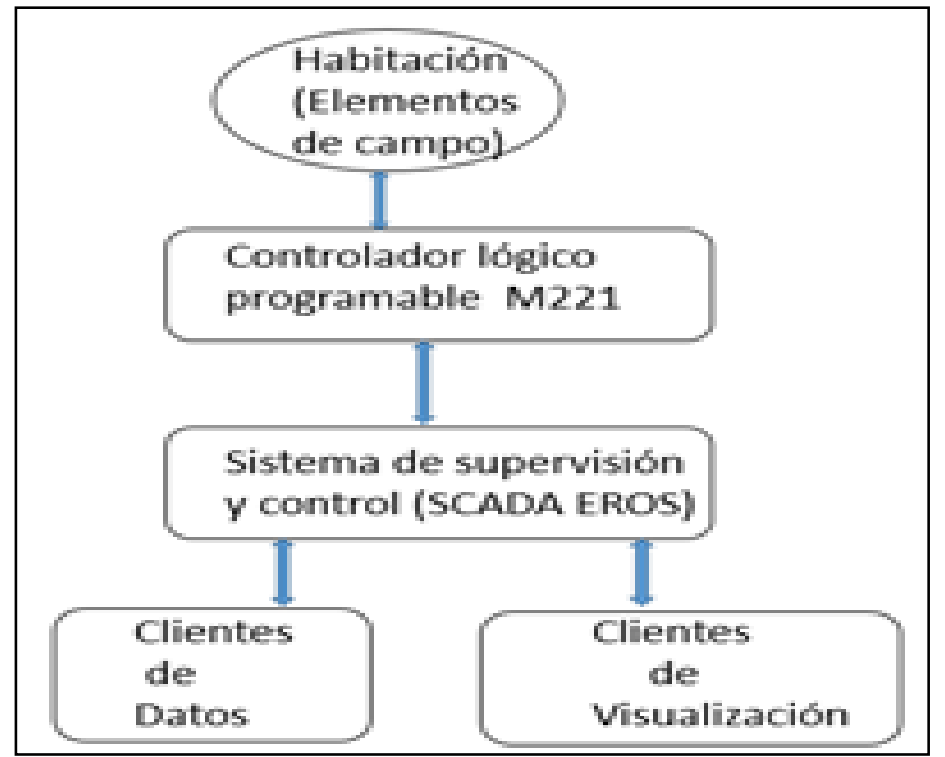

Figura 5: Partes del sistema SCADA.

Fuente: Los autores, (2018).

El SCADA se implementa en una computadora central, que sirve como HMI (Interfaz Hombre-Máquina), la cual controla toda la información proveniente de cada uno de los PLC conectados:

- Monitoreo y supervisión en tiempo real de la ocupación de la habitación: Condición de la habitación (habitación ocupada o libre), presencia de huésped (habitación en uso o habitación en no uso).

- Monitoreo en tiempo real del estado de las puertas y ventanas.

- Monitoreo y supervisión en tiempo real de la temperatura.

- $\quad$ Estado de alarma por detección de humo.

- Monitoreo y supervisión en tiempo real de la iluminación en habitaciones y pasillos.

- $\quad$ Establecimiento del punto de consigna de la temperatura en las habitaciones.

- El sistema de Control y de Supervisión (SCADA) estará centralizado y contará con tres niveles de dirección:

- $\quad$ - El primer nivel:

- Constituido por los elementos de campo (sensores y actuadores) que se encargan de enviar toda la información de los equipos tecnológicos y de recibir las señales de control de los niveles superiores. Ej: Válvulas de agua fría, termostatos de temperatura, sensores de presencia, módulos de contactores magnéticos de los interruptores automáticos de alumbrado, etc.

Para realizar las mediciones de las diferentes magnitudes físicas, eléctricas, mecánicas y otras que provienen de un medio exterior se utilizan dispositivos llamados sensores y transductores. Todos los elementos de campo utilizados son de la firma Schneider Electric.

Los sensores, considerados elementos transductores de entrada en un sistema domótico, permiten obtener los datos de los parámetros que se desean monitorear y/o controlar, realizando la conversión de magnitudes para transmitirla a la unidad encargada del procesamiento y control del estado de las variables a gestionar [6].

\section{III.4 DETECTORES DE PRESENCIA}

En las habitaciones se utilizan detectores de presencia ARGUS MTN 5510-1219 diseñados para cubrir la necesidad de conexión de la iluminación de una estancia donde se va a realizar una actividad, con un área de cobertura de $360^{\circ}$. Detectores tipo PIR que encienden la luz y la deja encendida hasta que detecta que ya no hay nadie en la habitación. Este se alimenta a 230VAC, $60 \mathrm{~Hz}$ y tiene un alcance de un radio de $7 \mathrm{~m}$. El dispositivo tiene salida a relé para detectar movimientos en función de la luminosidad, por ejemplo, para encender la iluminación. El tiempo de encendido puede ajustarse en el sensor de forma continua entre $10 \mathrm{~s}$ y $30 \mathrm{~min}$. El detector comprueba constantemente la luminosidad de la habitación y, si hay suficiente luz natural, apaga la luz artificial, aunque alguien se encuentre en la habitación, tiene un ajuste entre 10 y 1000 luxes. Su conexión al PLC es por cable UTP.

Para los pasillos se utilizan detectores PIR del Sistema ARGUS MTN550499 de presencia de empotrar con alimentación a $230 \mathrm{VAC}, 60 \mathrm{~Hz}$ compuesto por el cabezal sensor y un componente de potencia con cable fijo de $2.5 \mathrm{~m}$, el cual se enchufa al cabezal sensor. Posee un área de cobertura de $360^{\circ} \mathrm{y}$ un alcance de $4 \mathrm{~m}$ para una altura de montaje de $2.5 \mathrm{~m}$, cada cabezal sensor tiene 2 conectores hembra, por lo que se puede tender un cableado de paso. De este modo se pueden conectar un máximo de 8 cabezales sensores en un mismo componente de potencia (principio maestro-esclavo) con una distancia de $8 \mathrm{~m}$. Se puede ajustar la luminosidad entre 10 y 1000 luxes. Este detector se conecta al PLC de la habitación más cercana al cuarto local de automatización por piso. Su conexión al PLC es por medio de cable UTP.

\section{III.5 DETECTOR DE HUMO}

El detector de humo óptico CONNECT 230V MTN548520 con una alimentación a 230 VAC detecta a tiempo los incendios. Funciona según el principio demostrado de dispersión de la luz. Los emisores y receptores de luz están colocados en la cámara de medición de forma que el haz luminoso del emisor no puede llegar directamente al receptor. Primero llega al receptor la luz dispersa por las partículas flotantes (efecto Tyndall), y luego se convierte en señal eléctrica. Gracias a este modo de construcción, no necesita ningún preparado radioactivo. El detector de humo detecta los incendios con rapidez y avisa de ellos. Si entra humo en la cámara de medición, se activa la señal acústica incorporada.

\section{III.6 TERMOSTATO DE TEMPERATURA}

Para medir la temperatura se utilizarán termostatos de temperatura de tipo TC303-3A4DLMS De Luxe de la serie TC300. Este cuenta con un control basado en microprocesador y una gran pantalla LCD retroiluminada. Los modos de pantalla LCD incluyen el estado de funcionamiento (refrigeración, calefacción y ventilación), velocidad del ventilador, temperatura ambiente y punto de ajuste. Este se comunica con el autómata de la habitación a través del protocolo Modbus TCP/IP en tiempo real con cable UTP de $4 \times 0.75 \mathrm{~mm}^{2}$, vienen con la función de fallo de alarma incorporada para facilitar el mantenimiento.

Con una alimentación a 230VAC solo se activará el fan coil si hay presencia física del cliente habilitado y la temperatura del local es mayor que la predefinida como punto de consigna (set point), permitiendo fijar un punto de consigna de 
temperatura, encender y apagar el fan coil y el cierre y apertura de las válvulas de forma local y remota.

Se instala una sonda de temperatura en el conducto de retorno del fan coil para promediar el valor real de temperatura dentro de la habitación y obtener un mejor control de esta variable.

\section{III.7 CONTACTOS MAGNÉTICOS}

Con los contactos magnéticos marrones 663093 se detectarán puertas y ventanas abiertas para poner el clima en operación económica al igual que cuando no esté el huésped en la habitación, pero después de 10 minutos. En posición neutral el relé está cerrado o abierto en dependencia si el contacto es normalmente cerrado o abierto, la apertura del objeto custodiado está supervisada mediante la distancia entre el relé y el magnético. En cuanto el magnético se aleja de la parte del contacto, el campo magnético, que afecta al relé, se reduce hasta que se cierra o abre y da una señal.

\section{III.8 INTERFAZ DE COMUNICACIÓN ACTI 9 SMARTLINK}

La interface de comunicación inteligente Acti 9 Smartlink está diseñado para el control de los breakers termo magnéticos en las pizarras eléctricas. Es un protocolo universal y abierto que ofrece la posibilidad de comunicarse con el PLC por el conector RS 485 utilizando el Bus Modbus TCP/IP en conexión directa. $\mathrm{Su}$ montaje se puede realizar en rail DIN sin necesidad de suplementar espacio, posee 11 canales de entrada/salida y cada canal posee 2 entradas digitales a 24 VDC y una salida digital a 24 VDC, su alimentación es a $24 \mathrm{~V}$ DC. Los breakers termo magnéticos se deben usar con el dispositivo auxiliar de indicación iOF+SD24.

\section{III.9 CERRADURAS ELECTRÓNICAS RFID}

Con el fin de reducir los principales gastos energéticos del hotel se implementa un sistema con cerraduras electrónicas RFID (Identificación por Radiofrecuencia) a 13.56 Mhz para incrementar la seguridad y simplificar la gestión de la institución, ofreciendo muchas más prestaciones que la simple apertura y cierre de puertas.

Los sistemas RFID a $13.56 \mathrm{MHz}$ permiten la lectura/escritura del código de una tarjeta a corta distancia. No se requiere contacto con el lector, los códigos son leídos a distancia, de esta manera, se puede identificar y sin necesidad de contacto, con más comodidad, seguridad y durabilidad. El rango de lectura va de unos centímetros (proximidad) hasta varios metros, usando RFID activos, ideales para aplicaciones de trazabilidad y localización.

El funcionamiento es muy sencillo, el lector lanzará una petición a través de radiofrecuencia al chip que estará dentro del RFID. El mismo mandará una respuesta al lector, mostrando que es una persona autorizada. En este momento, la identificación se aprobará en tiempo real. Es un sistema de seguridad muy completo y realmente muy seguro donde el código es único.

$\mathrm{La}$ frecuencia $13.56 \mathrm{MHz}$ no solo ofrece una gran seguridad, sino que en las propias tarjetas se puede guardar mucha información, para más tarde ser transferida rápidamente. El software provisto junto con la cerradura permite ser usado por diversos operadores.

Las tarjetas se pueden usar por clientes, personal de limpieza, personal de mantenimiento, gerencia y en caso de emergencia.

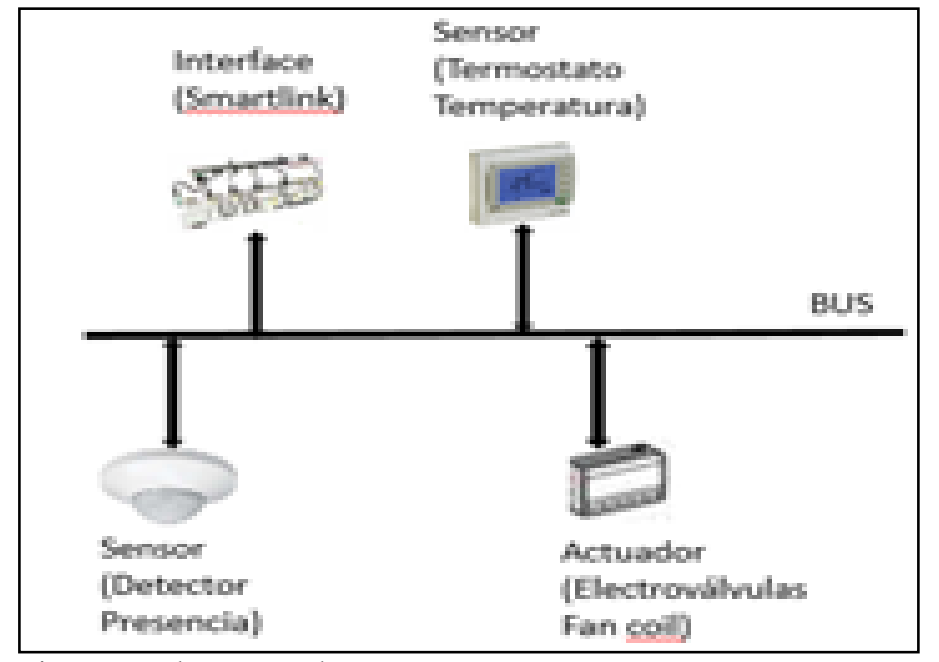

Figura 6: Elementos de campo.

Fuente: Los autores, (2018).

El segundo nivel:

- Se encargará de procesar toda la información del primer nivel. Tienen la posibilidad de comunicarse con controladores de su mismo nivel mediante Bus de datos (Modbus TCP/IP); y con el nivel superior. Estos controladores estarán distribuidos por todos los niveles habitacionales. Ej: Controladores Modicon M221.

Como sistema de control para leer y procesar los datos de los sensores se utilizan los controladores lógicos programables (PLC).

Los PLC están definidos por la "National Equipment Manufacturer's Asociation, (NEMA)" como: "aparatos electrónicos digitales que usan técnicas de programación de instrucciones almacenadas internamente para implementar funciones específicas tales como lógicas secuenciales, de temporización, de conteo y aritméticas para el control de máquinas y procesos".

A diferencia de una computadora de control el PLC no requiere de programación, ni depuración, ni técnicas de mantenimiento muy sofisticadas. Los PLCs, se usan para el control de secuencias [11].

Se utiliza el PLC tipo Modicon M221CE16R para las habitaciones y el SmartStruxure en la sala central de automatización.

El Modicon M221CE16R es un PLC que se utiliza en cada habitación y se configura como esclavo. La configuración, programación y puesta en funcionamiento se lleva a cabo con el software SoMachine Basic descrito en la guía SoMachine Basic Guía de funcionamiento. Se escogió el M221CE16R por su amplia variedad de potentes funciones, con una alimentación de 100-240VAC, 9 entradas/7 salidas lógicas y 2 entradas analógicas de 0 a 10V. Estos se conectan vía Ethernet TCP/IP a través de un conector RJ45 y par trenzado UTP con el switch del piso TCSESM163F2CSO de 14 puertos con entrada de fibra óptica en modo monomodo.

El PLC SmartStruxure supervisa y gestiona dispositivos del bus de campo. El software de Operación de Edificio EcoStruxureTM (antes conocido como software StruxureWare) mediante el cual se configura, programa y pone en funcionamiento, proporciona monitoreo integrado, control y gestión de energía, sistemas de climatización, iluminación y seguridad contra incendios. Es un sistema centralizado con 
escalabilidad desde un solo edificio hasta una empresa global. En instalaciones medianas y grandes, la funcionalidad se distribuye a través de múltiples funciones. Son PLC que se comunican a través de TCP/IP.

El PLC SmartStruxure, utilizado como estación de trabajo profesional, SW-STATION-PRO-O (que incluye editor gráfico, script y editores de programación de bloques de funciones), se configura como master en la sala central automatización, requiere del módulo $\mathrm{PS}-24 \mathrm{~V}$ que es una fuente de corriente directa que se alimenta a 24VAC o 24VDC y da a la salida 24VDC. Se comunica a través del conector Ethernet TCP/IP con el switch TCSESM163F2SCO de la sala central de automatización por medio del conector RJ 45 y par trenzado UTP.

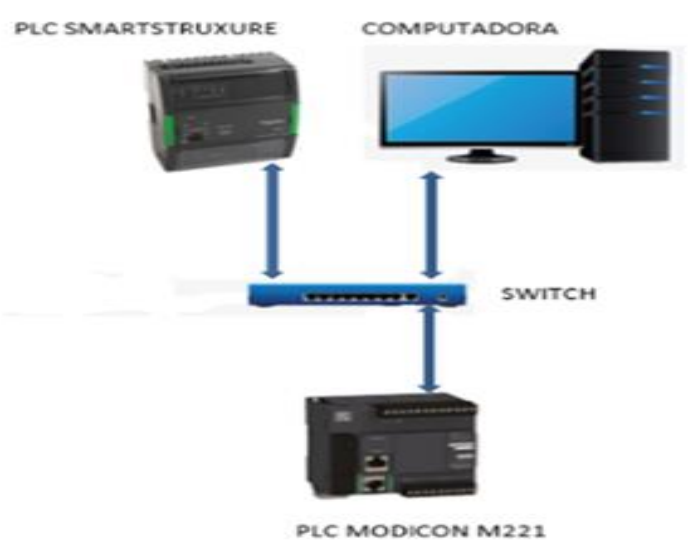

Figura 7: Conexión de los PLC al SCADA.

Fuente: Los autores, (2018).

En la tabla 1 se pueden observar las entradas y salidas al PLC Modicon M221.

Tabla 1: Entrada/salida a supervisar y/o controlar por PLC.

\begin{tabular}{|c|c|c|c|c|c|}
\hline Variables & ED & EA & so & SA & Observación \\
\hline Detector de presencia para iluminación & $\mathrm{x}$ & & & & \\
\hline Detector de humo & $x$ & & & & \\
\hline Termostato de temperatura digital & & & & & Bus MODBUS \\
\hline Contacto magnético puerta entrada & $x$ & & & & \\
\hline Contacto magnético ventana & $x$ & & & & \\
\hline Llave de entrada RFIO & $x$ & & & & \\
\hline $\begin{array}{l}\text { Sonda de temperatura en conducto de } \\
\text { retorno de fon coil. }\end{array}$ & & $\mathrm{x}$ & & & \\
\hline Acti 9 Smortink & & & & & Bus MODBUS \\
\hline Alarma al panel de corrientes debiles & & & $\mathrm{x}$ & & \\
\hline $\begin{array}{l}\text { Nivel bajo ventilador de fon soil } \\
\text { (Electrovilvuls) }\end{array}$ & & & $x$ & & \\
\hline $\begin{array}{l}\text { Nivel medio ventilador de fon ceil } \\
\text { (Electrovilivula) }\end{array}$ & & & $\mathrm{x}$ & & \\
\hline $\begin{array}{l}\text { Nivel alto ventilador de fon sell } \\
\text { (Electrovsilvuls) }\end{array}$ & & & $x$ & & \\
\hline Estado de habitación & & & $\mathrm{x}$ & & \\
\hline $\begin{array}{l}\text { ED: Entrada digital } \\
\text { EA: Entrada analógica } \\
\text { SD: Salida digital } \\
\text { SA: Salida analógica }\end{array}$ & & & & & \\
\hline
\end{tabular}

Fuente: Los autores, (2018).

- $\quad$ El tercer nivel:

Nivel de supervisión y control centralizado donde se recibe, mediante la red Ethernet de cableado estructurado (Protocolo TCP/IP), toda la información de los controladores programables de los procesos y equipos que controlan. En este se ubica una PC (computadora personal) para realizar esta acción.

\section{III.9 PANTALLA PROFESIONAL TÁCTIL}

En aras de brindar mayor comodidad y facilidad a los huéspedes se incorporan en las habitaciones pantallas profesionales táctiles interactivas Big Pad de Sharp de 60" soportadas a pared como la PN-L602B. Utilizan el sistema de detección de bloqueo infrarrojo propiedad de Sharp, que utiliza múltiples sensores infrarrojos para localizar la posición del dedo del usuario o el lápiz táctil. Estas pantallas permiten dibujar, escribir y manipular el contenido de la pantalla, por lo que brinda la comodidad de escritura en pantalla para mayor variedad profesional, es una herramienta que permite que los usuarios escriban en la pantalla Big Pad (hasta cuatro personas simultáneamente) a través de sus dispositivos utilizando los Pen Software (lápices táctiles) conectados de forma inalámbrica.

El control táctil permite a los usuarios cambiar páginas y ampliar o mover un objeto, hacer anotaciones a la vez que muestra texto, imágenes y notas escritas a mano y permiten comunicación bidireccional.

Se pueden enlazar a través de una Tableta (PC) con Android e iOS permitiendo lograr mayor información con el contenido de forma fácil e intuitiva.

Esta pantalla con una alimentación de 100-240VAC, $60 \mathrm{~Hz}$ se conecta por medio de la placa interfaz PN-ZB01 y un conector RJ45 a un enrutador y servidor que forman parte de una red LAN hasta la PC de Carpeta y Comercial permitiendo los siguientes servicios:

- Bienvenida.

- Matutino.

- Selección de lenguaje.

- Información del Hotel.

- Servicio de Mensajería.

- Servicio de Habitaciones.

- Selección de programas de TV.

Check out

- Otros.

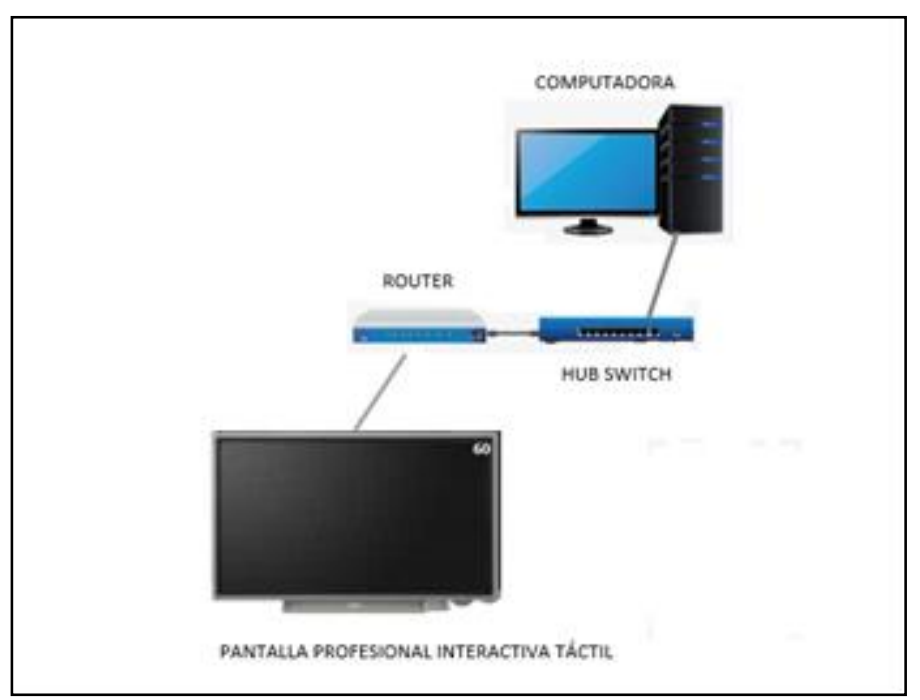

Figura 8: Pantalla profesional conectada a PC.

Fuente: Los autores, (2018).

Se instalarán registros de $100 \mathrm{~mm}$ x $100 \mathrm{~mm}$ x $55 \mathrm{~mm}$ en todas las habitaciones $y$ en los pasillos habitacionales, incluyendo los casetones registrables de los pasillos habitacionales para el cableado desde las bandejas hasta los paneles eléctricos de las habitaciones. 


\section{CONSIDERACIONES FINALES}

Con la utilización de las TIC y las herramientas necesarias para el diseño de un sistema inmótico en un hotel 5 estrellas plus se logra aumentar la eficiencia energética, el confort, la seguridad, mejorar las comunicaciones y la accesibilidad, así como el control y la gestión.

Para lograr la comunicación entre todos los dispositivos del sistema inmótico se utiliza la arquitectura de red distribuida, la cual, a través del bus enlaza todos los elementos del sistema y donde cada elemento tiene una dirección IP. Cuenta con una PC en la sala de control para supervisar y controlar las diferentes variables.

En el diseño se utiliza la red Ethernet TCP/IP y el protocolo de comunicación Modbus TCP/IP. Esta red transmite los datos en forma de paquete y cada nodo escucha dicha transmisión y verifica si está destinada a ese nodo; solo responde el nodo que corresponde al direccionamiento de destino. El protocolo Modbus TCP/IP permite desarrollar el formato de los mensajes que los diferentes elementos de control deben utilizar para entenderse unos con otros y que puedan intercambiar información de una manera segura y coherente.

Para el diseño del sistema de control supervisorio y adquisición de datos, SCADA, se utiliza el software profesional EROS, y el mismo estará centralizado y contará con tres niveles de dirección.

- En el primer nivel se encuentran los elementos de campo, sensores y actuadores, que se encargan de enviar toda la información y de recibir las señales de control de los niveles superiores.

- Dentro de las habitaciones se utilizan detectores PIR (Presencia Infrarrojo) de presencia ARGUS MTN 5510-1219, que presentan características que lo hacen idóneos para este tipo de sistemas, pues cuentan con un área de cobertura de $360^{\circ}$, tienen un alcance de $7 \mathrm{~m}$, presenta un ajuste entre 10 y 1000 luxes y su conexión al PLC es por cable UTP.

En los pasillos se utilizan detectores de presencia PIR del tipo ARGUS MTN550499 de empotrar. Los mismos poseen un área de cobertura de $360^{\circ}$ y un alcance de $4 \mathrm{~m}$ con una altura máxima de montaje de $2.5 \mathrm{~m}$. Pueden conectarse un máximo de 8 dispositivos sensores, principio maestro-esclavo, con una distancia de $8 \mathrm{~m}$.

El detector óptico de humo CONNECT 230V MTN548520 se utilizará dentro de las habitaciones. Funciona según el principio de dispersión de la luz. Los emisores y receptores de luz están colocados en la cámara de medición de forma que el haz luminoso del emisor no puede llegar directamente al receptor. Si llega el humo a la cámara de medición se activa la señal acústica incorporada.

Para medir la temperatura se utilizarán termostatos de tipo TC303-3A4DLMS De Luxe de la serie TC-300. Se comunica con el autómata de la habitación a través del protocolo MODBUS TCP/IP con cable UTP de $4 \times 0.75 \mathrm{~mm}^{2}$ y vienen con la función de fallo de alarma incorporada para facilitar el mantenimiento. Además, se instala una sonda de temperatura en el conducto de retorno del fan coil para promediar el valor real de temperatura dentro de la habitación y obtener un mejor control de esta variable.

- El segundo nivel se encargará de procesar toda la información del nivel anterior. Se utiliza el PLC Modicon M221 como esclavo para comunicar todos los elementos de campos y el PLC SmartStruxure como Maestro para controlar los dispositivos del bus de campo.
- El tercer nivel, de supervisión y control centralizado, recoge toda la información de los PLC del sistema mediante la red Ethernet de cableado estructurado (Protocolo TCP/IP). En este nivel se ubica una PC para realizar esta acción y mostrar el SCADA diseñado.

\section{REFERENCIAS}

[1] Percy Viego, Felipe; Cambra Díaz, Adrian; Cortiza Sardiñas, Rocío y Martínez Hernández, Tanayi. Edificios inteligentes. Consultado el 15 de febrero 2018. Disponible en: http://www.cubasolar.cu/biblioteca/Energia/Energia60/HTML/A rticulo09.html

[2] Tiscornia, Eduardo; De Felipe, Ariel. Domótica: La vivienda inteligente. Consultado el 15 de febrero 2018. Disponible http://www.palermo.edu/ingenieria/downloads/CyT3/CYT308.p df

[3] Bravo Santos, Crescencio. Un sistema de soporte al aprendizaje colaborativo del diseño domótico mediante herramientas de Modelado y Simulación. Tesis Doctoral. UNIVERSIDAD DE CASTILLA - LA MANCHA, Escuela Superior de Informática de Ciudad Real Departamento de Informática, Ciudad Real, 2002.

[4] Guido Miranda, Reyna; Villacrés Moreno, Raúl; Villamar Mendieta, Franklin. Diseño de un sistema domótico aplicado a una clínica de hemodiálisis. Tesis de grado. Guayaquil Ecuador. Año 2009.

[5] Junta de Andalucía. Estado del arte de las TIC aplicada a la edificación inteligente. Consejería de Economía, Innovación y Ciencia, octubre 2011.

[6] Henao Merchán, Oscar David. Hardware y software domótico. Universidad Pontifica Bolivariana, Escuela de Ingenierías, Facultad de Ingeniería eléctrica y electrónica, Medellin, 2006

[7] Mendiburu Díaz, Henry Antonio. AUTOMATIZACIÓN MEDIO AMBIENTAL. Aplicación de la automatización industrial $y$ el control de procesos, en la protección y conservación del medio ambiente. Lima, Perú. MMIII.]

[8] Núñez Álvarez, José Ricardo. Paquete tecnológico: Sistema de supervisión $y$ control utilizando protocolos de comunicación inalámbricas. Universidad de Oriente, Facultad de Ingeniería Eléctrica, Departamento de Control Automático, diciembre 2014.

[9] Observatorio Industrial del Sector de la Electrónica, Tecnologías de la Información y Telecomunicaciones, Redes de sensores. Aplicaciones para control automático de edificios, enero 2010.

[10] Carrero T, Dany N. Diseño de un sistema de control supervisorio y adquisición de datos (SCADA) para el monitoreo remoto de los sistemas de energía ininterrumpida (UPS) perteneciente al sistema eléctrico de una refinería en el país. Universidad de Carabobo, Facultad de Ingeniería, Escuela de Ingeniería Eléctrica, Departamento de Sistemas y Automáticas. Valencia - Venezuela. Valencia, abril de 2008. 
[11] Program Logic Control. Consultado el 12 de enero 2018. Disponible

www.oocities.org/gabrielordonez_ve/Unidades_Remotas_SCAD A.htm

[12] Ramírez, J. G. Diseño de un sistema inmótico en el Hotel Club Amigo Marea del Portillo en Pilón, Granma. Tesis en opción al Título de Ingeniero en Automática. Facultad de Ingeniería Eléctrica. Universidad de Oriente. Santiago de Cuba. Cuba. 2014.

[13] DOMODESK. (2015). A Fondo Inmótica. Consultado el 10 de febrero 2018. Disponible en: http://www.domodesk.com.

[14] Ortiz Cabrera, Mario Andrés. Optimización del sistema inmótico en el hotel RENAISSNCE de Barcelona. Trabajo de fin de Master, Escola Politécnica Superior d’Edificació de Barcelona, marzo 2011.

[15] Carbonell Mestre, Danilo Mariano. Diseño de un sistema inmótico en el bloque habitacional del hotel Plaza de Santiago de Cuba. Tesis presentada en opción al Título académico de Master en Ciencias en Automática, Universidad de Oriente, Santiago de Cuba 2018.

[16] LUGLI, Alexandre Baratella; SANTOS, Max Mauro Dias. Sistemas fieldbus para automação industrial:deviceNet, CANopen, SDS e Ethernet. Erica 2009.

[17] LUGLI, Alexandre Baratella; SANTOS, Max Mauro Dias. Redes Industriais para Automação Industrial: ASI, PROFIBUS e PROFINET. Erica 2010.

[18] Stuart G. McCrady Designing SCADA Application Software. Elsevier.USA. 2013.

[19] Krutz, Ronald L. Securing SCADA Systems. Wiley Publishing, Inc., Indianapolis, Indiana. USA. 2006

[20] Kurouse, Ross. Redes de Computadores y la Internet. Un enfoque top-down. 6ta edición. Editorial Pearson 2014.

[21] Behrouz, A. Forouzan. Transmisión de datos y redes de comunicaciones. Segunda edición. McGraw-Hill España. 2002.

[22] Aguilar, José; Rios Bolivar, Addison; Hidrobo, Francisco; Cerrada, Mariela. Sistemas MultiAgentes y sus Aplicaciones en Automatización Industrial. 2da Edición. ULA, Mérida, Venezuela, 2013. 